\title{
Guillan-Barré Syndrome after First Vaccination Dose against COVID-19: Case Report
}

\author{
Daniel Čenščák ${ }^{1}$, Leoš Ungermann², Ivana Štětkářová ${ }^{3}$, Edvard Ehler ${ }^{4, *}$
}

\begin{abstract}
A number of neurological complications have been reported after the administration of flu vaccine, including Guillain-Barré syndrome (GBS), especially after vaccination against swine flu. Only facial nerve neuropathy has thus far been reported after vaccination against COVID-19. More recently, there was a case of an elderly woman with GBS. In our report, we describe a case of a 42-year-old, previously almost healthy male who developed sensory symptoms 14 days after the first dose of Pfizer vaccine. One week later, the patient developed right facial nerve palsy and lower limb weakness and was no longer able to walk. Albuminocytological dissociation was detected in the cerebrospinal fluid, and there were inflammatory radicular changes in MRI scans of the lumbosacral spine. EMG indicated significant demyelinating polyradiculoneuritis and no antibodies against gangliosides were demonstrated. A 5-day course of immunoglobulins at a dose of $2 \mathrm{~g} / \mathrm{kg}$ lead to a significant improvement and the patient was soon able to walk. In conclusion, we report a case of Guillan-Barré syndrome after COVID-19 vaccine in a young patient with a rapid diagnosis and prompt administration of immunoglobulins.
\end{abstract}

\section{KEYWORDS}

vaccines; polyradiculoneuritis; ataxia; nerve conduction studies; COVID-19; Guillain-Barré syndrome; immunoglobulins

AUTHOR AFFILIATIONS

${ }^{1}$ Department of Neurology, District Hospital Pardubice, Czech Republic

2 Department of Radiology, Faculty of Health-Care study, Pardubice University, District Hospital Pardubice, Czech Republic

${ }^{3}$ Department of Neurology, 3rd Medical Faculty Charles University, Prague, and University Hospital Královské Vinohrady, Prague, Czech Republic

${ }^{4}$ Department of Neurology, Faculty of Health-Care Study, Pardubice University, District Hospital Pardubice, Czech Republic

* Corresponding author: Department of Neurology, Faculty of Health-Care Study, Pardubice University, District Hospital Pardubice, Czech Republic; e-mail: edvard.ehler@nempk.cz 


\section{INTRODUCTION}

Many vaccines can provoke autoimmune diseases, including GBS (Guillain-Barré syndrome). Flu vaccines, especially for swine flu, lead to multiple cases of vaccination-induced GBS, polyneuritis cranialis and other forms of peripheral or autonomic neuropathy (1). Facial nerve neuropathies and other peripheral nerve disorders have thus far been described in temporal occurrence after COVID-19 vaccination (2). GBS has been reported in only one 82-year-old woman after the mRNA vaccine (3). Later on some other papers were published on GBS and vaccines, which confirmed only temporal occurrence and not causality (4-6). In our report, we present the next case of a young 42-year-old male who also developed autoimmune inflammation of peripheral nerves (GBS) after the first vaccination dose against COVID-19 (Comirnaty, Pfizer), with a favourable course.

\section{CASE REPORT}

A 42-year-old male developed paraesthesia of the soles of his feet 14 days after the first vaccine dose against COVID-19 (mRNA vaccine, Comirnaty, Pfizer). The patient works as a nurse in a retirement home, he has been managed for bronchial asthma (budesonium, ipraproium, fenoterol inhalation twice a day) for 8 years.

Paraesthesia worsened the next day and spread to his hands as well. After a week, the patient developed gait problems; he had unsteady gait and weak knees. After 3 more days, he started to complain of right-sided mimic muscle weakness and lumbalgia. He was admitted to our neurological department 18 days after the onset of neurological problems (32 days after the first dose of vaccine).

In the clinical finding, there was right-side mimic muscle weakening, lagophthalmos on the right side up to 2 $\mathrm{mm}$, the philtrum was mildly pulled to the right side, there were no taste disturbances, he was ameningeal, was able to raise his head while lying on his back, the exteroceptive reflexes of the abdominal muscles were symmetric, handgrip strength $45 \mathrm{kPa}$ at the right side, $40 \mathrm{kPa}$ at the left side (median value per 42 years - $141.7 \mathrm{kPa}$ ), slight weakening in the elevation of the right arm, reflexes C5-8 minimal, L2-S2 not elicitable, lower limbs with a slow descent from rectangle elevation while lying on his back within 6 seconds on both sides, hypoesthesia $10 \mathrm{~cm}$ above the wrist and from the middle of the shanks for thermal and tactile qual-
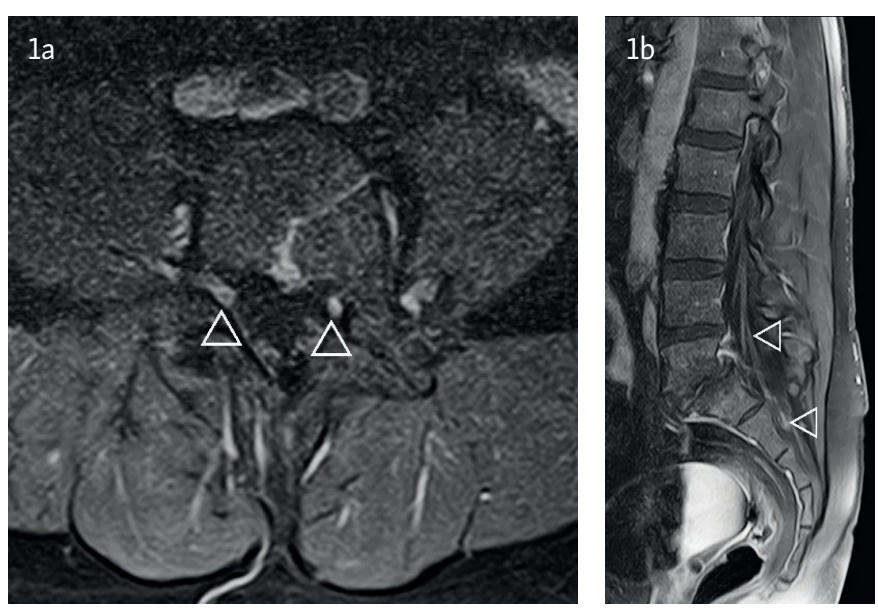

Fig. 1 In the axial (1a) and sagittal (1b) T1 weighted images after gadolinium there is mild thickening and enhancement of spinal roots in lumbosacral region.

ities, vibration sense $6 / 8$ on both sides. The patient was able to take 3-4 steps leaning on the furniture with ataxia and knees buckling.

Spirometry in a sitting position was performed (FVC (forced ventilation capacity): 0.62 l, FEV1 (forced expiratory volume in 1 second): 0.461 ), cerebrospinal fluid was collected, detecting albuminocytological dissociation (proteins $2.24 \mathrm{~g} / \mathrm{l}, 6$ leukocytes - all mononuclears), MRI of LS spine with post-contrast signal increase in the roots of cauda equina (Figures la and 1b). Antigen and PCR COVID-19 tests were repeatedly negative. Anti-ganglioside antibodies have not been detected.

Autonomic functions (handgrip test and Valsalva test) indicated a disorder of the autonomic nervous system with a decrease of heart rate variability.

Nerve conduction studies (NCS) and electromyography (EMG) examinations were performed immediately after admission to the neurological department, the results of which are presented in Table 1. Sensitive nerve action potentials (SNAP) were not elicitable in the lower limbs and low amplitude of SNAP $(2.6 \mathrm{uV})$ was found in the upper limbs (median nerve) with a significant reduction in the conduction velocity through sensitive fibres $(\mathrm{SCV}=18.2 \mathrm{~m} / \mathrm{s})$. Acute inflammatory demyelinating polyneuropathy (AIDP) with significant involvement of motor and sensitive fibres was confirmed based on the clinical findings and NCS/EMG examinations. NCS pointed to motor impairment both in the distal (DML - distal motor latency) and in the proximal section (F-waves) of the peripheral nervous system.

Tab. 1 Results of EMG examination at admission.

\begin{tabular}{|l|c|c|c|c|c|c|}
\hline Nerve (muscle) & DML (ms) & A-CMAP $(\mathbf{m V})$ & d-CMAP $(\mathbf{m s})$ & MCV & F-lat (ms) & F-wave persistence \\
\hline Median (APB) & $9.20 / 13.70$ & $2.6 / 2.6$ & 12.10 & 39.50 & 46.3 & $70 \%$ \\
\hline Ulnar (ADM) & $5.60 / 10.45$ & $2.9 / 1.9$ & 11.30 & 41.20 & 39.5 & $60 \%$ \\
\hline Peroneal (EDB) & $7.45 / 16.30$ & $2.6 / 2.2$ & 9.15 & 46.30 & 71.2 & $20 \%$ \\
\hline Tibial (AH) & $8.10 / 16.30$ & $0.7 / 0.2$ & 19.35 & 54.90 & 84.4 & $100 \%$ \\
\hline
\end{tabular}

Note: $\mathrm{DML}=$ distal motor latency, $\mathrm{A}-\mathrm{CMAP}=$ amplitude of compound muscle action potential, $\mathrm{d}-\mathrm{CMAP}$ duration of compound muscle action potential, $M C V=$ motor conduction velocity, $\mathrm{F}$-lat $=\mathrm{F}$ wave latency, $\mathrm{APB}=$ abductor pollicis brevis muscle, $\mathrm{ADM}=\mathrm{abductor}$ digiti minimi muscle, $E D B=$ extensor digitorum brevis muscle, $\mathrm{AH}=$ abductor hallucis muscle. 
The treatment started with intravenous administration of immunoglobulins ( $2 \mathrm{~g} / \mathrm{kg}$ over 5 days, at a total dose of $140 \mathrm{~g}$ ) on the day of admission. Paraesthesia in the limbs already improved during the treatment, only partially thus far, but gait and handgrip strength improved significantly. The patient was transferred to a rehabilitation institution, able to walk with minimal assistance (leaning on furniture), mild facial weakness remained, but without lagophthalmos. He was able to close his eyes actively and smile. There were no problems with breathing and swallowing. Vigorimetry (55 and $54 \mathrm{kPa}$ ) and spirometry (FVC 3.73 1, FEV1 1.62 1) parameters improved.

\section{DISCUSSION}

About $70 \%$ of patients with GBS have a clear trigger in the history (infection, surgery, stress, vaccination). Swineflu vaccination has led to individual GBS cases (1). Facial nerve palsy and later other peripheral nerv disorders were detected in a individuals after COVID-19 vaccination (2). It was not until February 2021 that a case of an 82-year-old woman who developed leg aches within a week of the first dose of vaccine (Pfizer) was published, followed by lower limb weakness the next week, leading to a fall and admission to neurology. Only hyperproteinorachia (0.88 g/l) was found in the cerebrospinal fluid. MRI of the LS spine detected root enhancement after gadolinium administration. IVIG administration lead to slow improvement of lower limb mobility and the patient regained gait ability (3). We are presenting the second case - a young male patient with GBS after COVID-19 vaccination. Fortunately, his problems regressed very quickly after immunomodulatory treatment. Our patient also had significant sensory disturbances and lower limb ataxia combined with mimic muscle weakness on the right side, inflammatory root changes in the lumbar region in MRI scans, and a closer examination revealed autonomic dysfunction (handgrip, Valsalva test). Anti-ganglioside antibodies were not detected. The clinical condition of this patient rapidly improved after the administration of intravenous immunoglobulins.

GBS, including its focal forms, may develop during COVID-19 infection (8). GBS has been published in 72 patients, presenting with sensory dysfunction in the lower limbs, ascending forms with upper limb involvement, either alone or in combination with paraparesis or tetraparesis (2). Ataxia was common, cranial nerve lesions occurred in $50 \%$ and dysphagia in $24 \%$ cases. About $36 \%$ of patients had respiratory muscle weakness (6). Autonomic dysfunction was uncommon in these patients. Peak clinical disability occurred 4 days after admission to hospital. EMG detected a demyelinating form of GBS in $77.4 \%$. The axonal form of GBS occurred in $14.5 \%$; the remainder were mixed forms (2). The clinical findings in patients with COVID-19 who develop GBS are similar, but the pathophysiological mechanisms are different. The absence of anti-ganglioside antibodies was unusual for patients with GBS prior to the COVID-19 pandemic. Patients with COVID-19-related GBS have typically more severe sensory impairment, less frequent involvement of the cranial nerves, marked ataxia, dysphagia is present in 50\%, and respiratory symptoms in $36 \%$ of patients. Autonomic symptoms are less common, and radicular gadolinium enhancement in lumbar region MRI scans is also rare. Our patient presented with peripheral quadriparesis with sensory disturbances and marked ataxia. EMG detected a demyelinating type of the disease. We proved the involvement of autonomic fibres was evident. An additional MRI scan was performed, detecting enhancement in inflamed and thickened roots in the lumbar region. The clinical findings improved rapidly and significantly after acute immunoglobulin treatment. Our patient differed from typical GBS forms, including post-COVID-19 GBS, since he presented with marked ataxia, absence of anti-ganglioside antibodies, and rapid clinical improvement after the administration of immunoglobulins.

Treatment of GBS is the same in patients with COVID-19 as in patients without COVID-19. Plasmapheresis provides the advantage of removing antibodies and cytokines. Nevertheless, the better tolerated administration of an immunoglobulin, which is also more comfortable for the patient, is preferred. Antibodies against COVID-19 could be included in current formulations.

There were some papers describing GBS after administration of vaccines - COVID-19 and influenza (9-12). Ogbebor et al. (4) described a case of GBS after vaccine administration against COVID-19. The first symptoms of GBS occurred the next day after first dose of vaccine (Pfizer). If the vaccine was the trigger, the symptoms developed 1-4 weeks after the trigger. The authors emphasise that temporal association should not be translated into causality. Patel et al. (5) presented a 37-year old man who developed GBS with ascending muscle weakness 3 weeks after the first dose of vaccine. Treatment with immunoglobulins lead to slow improvement. The authors suggested that GBS after Covid-19 vaccine is an extremely rarely reported side effect. Lunn et al. (13) looked at the development of GBS after vaccines. Approximately 1 billion people will be vaccinated, and an expert would expect 17,000 cases of GBS to occur sporadically per annum, of which 1,962 would occur in any 6-week period. It is therefore inevitable that many thousands of sporadic cases of GBS caused by other factors will appear temporally associated with COVID-19 vaccination. But this cannot be considered causal. Li et al. (14) presented a big multi-national network cohort study with potential incidence rates of potential adverse events of special interest. The calculated incidence rates are stratified by age, sex and database. Patients with GBS are on the lists published by regulators.

Only sporadic cases of GBS after administration of COVID-19 vaccines are published. According to larger epidemiological studies and meta-analysis of vaccines and development of GBS, we are convinced that vaccination against COVID-19 is not the cause of acute polyradiculoneuritis development, but there is only temporal association. A sporadic occurrence of GBS is in temporal association with vaccine administration. 


\section{REFERENCES}

1. Hampton LM, Aggarwal R, Evans SJW, Las B. General determination of causation between Covid-19 vaccines and possible adverse events. Vaccine 2021;39: 1478-80.

2. Abu-Rumeileh S, Abdelhak A, Foschi M, Tuman H, Otto M. Guillain-Barré syndrom spectrum associated with COVID-19: an up-todate systematic review of 73 cases. J Neurol 2021; 268(4): 1133-70.

3. Waheed S, Bayas A, Hindi F, Rizvi Z, Espinosa PS. Neurological Complications of COVID-19: Guillain-Barre Syndrome Following Pfizer COVID-19 Vaccine. Cureus 2021; 13(2): e13426.

4. Ogbebor O, Seth H, Min Z, Bhanot N. Guillain-Barré syndrome following the first dose of SARS-CoV-2 vaccine: A temporal occurrence, not a causal association. IDCases 2021; 24: e01143.

5. Patel SU, Khurram R, Lakhani A, Quirk B. Guillain-Barre syndrome following the first dose of the chimpanzee adenovirus-vectored COVID-19 vaccine, ChAdOx1. BMJ Case Rep 2021; 14(4), e242956.

6. Márquez Loza AM, Holroyd KB, Johnson SA, Pilgrim DM, Amato AA. Guillain- Barré Syndrome in the Placebo and Active Arms of a COVID-19 Vaccine Clinical Trial: Temporal Associations Do Not Imply Causality. Neurology 2021: 10.1212/WNL.0000000000011881.

7. Merkies I. Assessing grip strength in healthy individuals and patients with immune-mediated polyneuropathies. Muscle Nerve 2000; 23: 1393-401.
8. Dufour C, Co TM, Liu A. GM1 ganglioside antibody and COVD-19 related Guillain Barre Syndrome: A case report, systemic review and implication for vaccine development. Brain Behav Immun Health 2021; 12: 100203.

9. Park YS, Lee KJ, Kim SW, Kim KM, Su BC. Clinical features of post-vaccination Guillain-Barré Syndrom in Korea. J Korean Med Sci 2017; 32: 1154-9.

10. Soni R, Heindl SE, Wiltshire DA, Vahora IS, Khan S. Antigenic variability a potential factor in assessing the relationship between Guillain Barré syndrome and influenza vaccine - up to date literature review. Cureus 2020; 12(9): e10208.

11. Dotan A, Muller S, Kanduc D, David P, Halpert G, Shoenfeld Y. The SARS-CoV-2 as an instrumental trigger of autoimmunity. Autoimmune Rev 2021; 20(4): 102792.

12. Kochkar S, Salmon DA. Planning for COVID-19 vaccines safety surveillance. Vaccine 2020; 38(40): 6194-8.

13. Lunn MP, Cornblath DR, Jacobs BC, et al. COVID-19 vaccine and Guillain-Barré syndrome: let's not leap to associations. Brain 2021; 144(2): 357-60.

14. Li X, Ostropolets A, Makadia R, et al. Characterizing the incidence of adverse events of special interest for COVID-19 vaccines across eight countries: a multinational network cohort study. Preprint. medRxiv $2021 ; 2021.03 .25 .21254315$. 